\title{
Notre Axel,
} noyau dur de la Path Mol

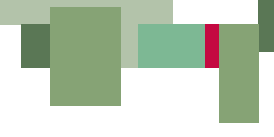

Arnold Munnich

Institut des maladies génétiques, Imagine, Hôpital

Necker-enfants malades et université de Paris

$>$ Axel est un diamant aux multiples facettes: ses élèves, ses collègues, ses amis en sont autant de facettes différentes. Nous avons tous eu avec lui une relation riche, privilégiée, singulière, passionnée, parfois tumultueuse. À chacun de nous «son Axel ». Notre Axel est venu de Beaujon dans les années 1970, à l'initiative de Jean-Claude Dreyfus, pour explorer à Cochin les bases moléculaires des anémies hémolytiques héréditaires, dans ce qui allait devenir l'Institut de pathologie moléculaire, «la Path Mol ». Invités par Robert Debré à le rejoindre après la guerre, les fondateurs de la Path Mol, Georges et Fanny Schapira, Jean-Claude Dreyfus et Jacques Kruh ont été froidement accueillis à Necker, malgré les terribles épreuves qu'ils avaient endurées et le courage dont ils avaient fait preuve. Ils y ont pourtant fait des travaux remarquables et de grandes découvertes, comme l'élévation des enzymes musculaires dans le sérum des femmes conductrices de myopathie de Duchenne. Mais on pardonne tout sauf le succès et les mandarins locaux de l'époque ne jugèrent pas opportun de leur réserver la place qu'ils méritaient dans le CHU Necker, en construction en 1968. Georges Schapira et son groupe, très soutenus par Jacques Monod, Prix Nobel 1965, furent bien mieux accueillis au CHU Cochin.

Les péchés d'orgueil se payent cher et pour longtemps. "Les malades à Necker et à Saint Vincent de Paul, la biologie à Cochin »: le schisme était consommé, le rêve de l'unité de lieu s'était envolé pour 40 ans. La contrepartie de cette erreur historique, de cette alliance ratée par Necker, ce fut la réussite de la «Path Mol » à Cochin. Réunion magique aux $4^{\circ}$ et $5^{\circ}$ étages du CHU Cochin de trois unités Inserm et d'un laboratoire de biologie hospitalière, sans consultation ni malades certes, mais formant une masse critique exceptionnelle de chercheurs et de biologistes hospitalo-universitaires, qui allait devenir un lieu de recherche, de formation, d'effervescence tout à la fois intellectuelle et politique, unique dans une France en assez bonne santé économique pour accompagner la vision des fondateurs de la Path Mol.

Après avoir ceint son tablier sur sa blouse blanche, Jean-Claude Dreyfus, vouté, les mains jointes dans le dos, arpentait chaque matin de long en large les couloirs de son unité, tantôt seul et pensif, tantôt devisant aux côtés de Livia Poenaru sur les enzymopathies lysosomiales, de Jean-Claude Kaplan et Dominique Labie sur les hémoglobinopathies, de Dominique Daegelen sur les myopathies métaboliques, de Claudine Junien sur les prédispositions cytogénétiques aux cancers, de Fanny Schapira et Annette Weber sur la fructosémie héréditaire.

Mais il fallait un sursaut, un souffle nouveau, une impulsion, une ambition nouvelle pour passer de la biochimie des protéines à la génétique moléculaire humaine. D’autant qu'au même moment, certains émules des pasteuriens prétendaient qu'il était «encore trop tôt pour étudier la génétique des maladies humaines à la Path Mol », et qu'il fallait « encore travailler sur les systèmes modèles ». Jean Rosa m'a confié qu'il venait «nuitamment », dans le plus grand secret, pour faire les électrophorèses des hémoglobinopathies. Visionnaire, Jean-Claude Dreyfus eut alors le trait de génie de convaincre Axel de le rejoindre, avec Joëlle Marie, brillante étudiante en thèse.

Je n'ai pas connu les premiers pas bondissants d'Axel à la Path Mol en 1975. En revanche, je me souviens parfaitement de l'imposante délégation de l'unité Inserm de J.-C. Dreyfus au colloque de la FEBS (Federation of European Biochemical Societies) à Dresde en 1977, bien avant la chute du Mur, comme je me souviens de l'éblouissante communication d'Axel sur les déficits en pyruvate kinase. Axel apportait de la méthode et de la rigueur à une spécialité médicale qui en manquait encore singulièrement et posait enfin les problèmes des maladies humaines en termes scientifiques, dans un anglais tout à fait correct, là où aucun de mes patrons n'auraient pu en faire autant. Axel avait l'assurance, le langage corporel d'un jeune homme ambitieux, non pour lui mais pour la cause qu'il allait servir toute sa vie, sans arrogance mais avec détermination: l'excellence de la médecine moléculaire. Inoubliable congrès où engagement scientifique et engagement militant - quasiment identitaire - semblaient ne plus faire qu'un. Au sortir de ce colloque, Jean-Claude Dreyfus devait me confier qu'il restait «pour quelques jours de vacances supplémentaires dans ce pays béni des Dieux qu'est l'Allemagne de l'Est ».

Avec une énergie et une générosité infatigable, Axel et Joelle ont formé, ensemble, toute une génération de jeunes thésards, post-doc, médecins, pharmaciens français et étrangers à l'étude de l'expression des gènes, de la régulation de la transcription, puis à la construction 
de banques et au clonage génomique et des ADNc. De la traduction in vitro des ARN messagers à la purification des produits de traduction sur mini-colonnes d'affinité, ils ont ouvert la voie à l'étude des maladies de la glycolyse, de la glycogénolyse, de la néoglucogenèse. Dans le mythique «labo 4009 », composé de quatre unités, accueillant chacune trois étudiants, Axel passait dix fois par jour, savait tout ce que faisait chacun. Nous guettant à la sortie de l'ascenseur qui desservait le laboratoire photographique, il interprétait nos autoradiographies dégoulinantes, avant même qu'on ait eu le temps de les analyser.

Arrivé au labo à l'aube, Axel avait lu la bibliographie pour tout le monde avant notre arrivée et parsemait les murs du couloir de post-it de couleurs, signalant les nouveautés conceptuelles et technologiques. Rédigeant lui-même les protocoles que nous nous passions de main en main, Axel se piquait de maîtriser sur le bout des doigts toutes les techniques qu'il nous faisait appliquer. II répétait avec une éloquence ponctuée d'imparfait du subjonctif : «je n'ai jamais pensé qu'il fût possible d'exiger de quiconque ce qu'on est incapable de réaliser soi-même ».

Mais, très réservé et peu enclin à l'enthousiasme communicatif d'Axel, J.-C. Dreyfus en demandait toujours plus et ne s'en cachait pas. "Il faudra bien en arriver un jour au clonage des ADNc et à l'identification des mutations! ». Claude Besmond, Marie-Pierre Simon, Georges Uzan, Michel Raymondjean, Pascal Maire construisirent les premières banques d'expression à Cochin, les criblèrent au moyen d'anticorps spécifiques et identifièrent alors les premiers clones $A D N C$ de pyruvate kinase $L$ et $M$, d'aldolase $A$ et $B$ et les premières mutations responsables des maladies qu'ils étudiaient. La pathologie moléculaire du gène en France était née simultanément à Cochin, à Pasteur (Jean Weissenbach et Christine Petit) et à Strasbourg (Igbmc, Jean-Louis Mandel).

Grâce à Axel, la Path Mol s'est imposée comme un centre de ressources où on venait «cloner son gène». Au risque d'une

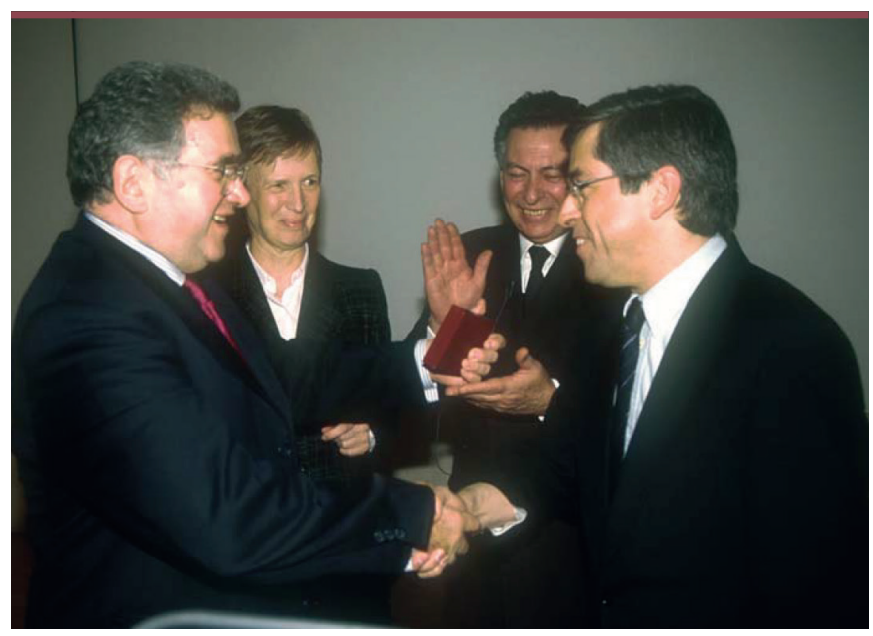

Arnold Munnich, lauréat du Grand Prix Inserm 2000, en présence de Claude Allègre (ministre de l'Éducation nationale, de la Recherche et de la Technologie), Dominique Meyer (présidente du conseil d'administration), Claude Griscelli (directeur général de l'Inserm). certaine dispersion, Axel ne disait presque jamais non, et trouvait toujours une excellente raison de dire oui. À la Path Mol sont venus se former comme nulle part ailleurs des générations d'étudiants désireux de s'initier aux principes et aux techniques de la biologie moléculaire et de se former «à et par la recherche» pour reprendre la formule de Philippe Lazar. Les séminaires bibliographiques du jeudi étaient une véritable préfiguration du défi suivant que devait se lancer Axel à lui-même : médecine/sciences. Axel n'avait pas le statut d'enseignant parce qu'il a toujours refusé d'être nommé professeur d'université. Cela aurait-il été une trahison à ses propres yeux? Peut-être. II n'en reste pas moins qu'Axel a été aussi le meilleur de tous nos enseignants. Si, comme l'écrit Montaigne: "l'élève n'est pas un vase qu'on remplit mais un feu qu'on allume », alors Axel a été un pyromane de génie pour beaucoup d'entre nous. Passionnant et passionné, affectueux et séducteur, fidèle et imprévisible, Axel a profondément aimé la science et nous I'a faite aimer. II a fait de beaucoup d'entre nous ce que nous sommes aujourd'hui. Merci Axel! $\diamond$

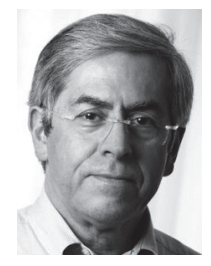

Arnold

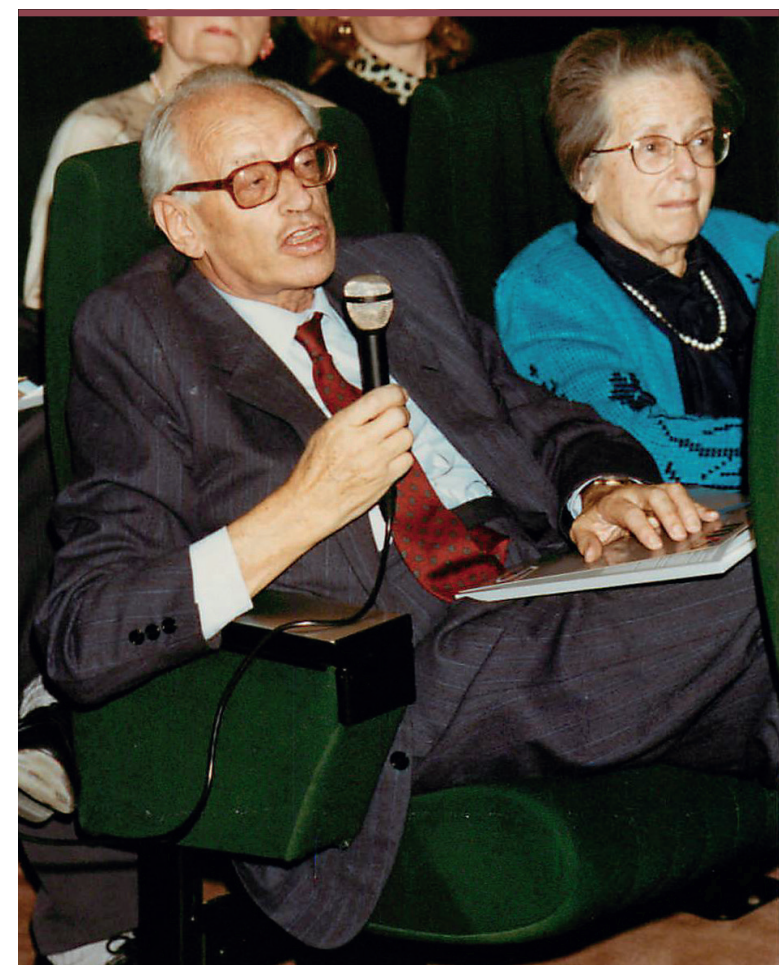

Jean-Claude Dreyfus et son épouse pendant les cessions du colloque du $X^{e}$ anniversaire. 